\title{
ELECTROOXIDATION OF DNA AT GLASSY CARBON ELECTRODES MODIFIED WITH MULTI-WALLED CARBON NANOTUBES WITH DIFFERENT OXIDATION DEGREE
}

\author{
PAULINA CAÑETE-ROSALES, ALEJANDRO ÁLVAREZ-LUEJE, SOLEDAD BOLLO*
}

Laboratorio de Bioelectroquímica, Facultad de Ciencias Químicas y Farmacéuticas, Universidad de Chile, Santiago, Chile.

\begin{abstract}
For the first time, the study of the effect of length and degree of oxidation of multi-walled carbon nanotubes (CNTs) on the electroanalytical DNA sensing properties of modified glassy carbon electrodes (GCEs) is reported. GCE/CNT electrodes were prepared using chitosan (CHI) as a dispersing agent. Short (S-NCs) and long (L-NCs) carbon nanotubes, as well as unoxidized and 3 or 6 hour oxidized samples were used. Cyclic voltammetry indicated that S-NCs exhibited larger capacitance values than L-NCs, and that these values increased when the CNTs were oxidized. Using Scanning Electrochemical Microscopy (SECM), an increase in the surface homogeneity was determined when oxidized CNTs were used as electrode modifiers. dsDNA electrooxidation showed the highest response using GCEs/CHI-L-NCs, and a clear decrease in the current intensity was observed when the oxidation time was increased. This result correlates with the higher BET surface area of L-NCs. In conclusion, the electrochemical performance of GCE/CNT electrodes against DNA increase when the length of CNTs is higher and decrease at higher oxidation state.
\end{abstract}

Key words: Carbon nanotubes, oxidation, Chitosan, glassy carbon electrode, dsDNA.

\section{INTRODUCTION}

Multi-walled carbon nanotubes (CNTs) have been used extensively in electrochemical studies due to their electrocatalytic effects on redox processes of different analytes ${ }^{1,2}$. These effects have mainly been attributed to their great surfaces for adsorption, an improvement in electron transfer and the presence of impurities such as $\mathrm{Fe}$, among others. In this context, many works related to CNT-based electrochemical sensors for bioanalytical applications have been published, finding use in clinical chemistry, food quality and control, drug analysis, waste water treatment and bioprocessing ${ }^{3-5}$.

Despite all of the electroanalytical advantages from CNTs, a major experimental drawback is their insolubility in all solvents, which makes their use as surface electrode modifiers difficult. Different approaches have been proposed to solve this problem, such as the use of surfactants, polymers and biomolecules ${ }^{6-9}$, functionalizing the sidewalls or covalent binding ${ }^{10-13}$. However, many works have stated the necessity of oxidizing CNTs prior to electrode modification. Studies have shown that, in general, oxidation was performed to eliminate metallic impurities ${ }^{14}$, improve their solubility in different media ${ }^{15}$ or facilitate further chemical modifications ${ }^{16,17}$. Despite all of this, there are few studies devoted to a systematic evaluation of the effects of oxidation treatments with regard to the electroanalytical properties of CNT-modified electrodes and how this treatment can affect the response of the electrode to the adsorption process of a particular analyte. In 2002, Li et al. ${ }^{18}$ reported an electrochemical study of carbon nanotube ensemble electrodes and obtained information about the capacitive background and electron transfer rate from the faradaic reaction of ferricyanide. They concluded that the apparent electron transfer rate correlated to both the area of the exposed side-walls and the density of graphite edge plane-like defects. Later, Lawrence et al. ${ }^{19}$ performed a comparison of the electrochemical reactivity of electrodes modified with CNTs from different sources. They showed a dependence between the electrocatalytic activity, background current and electroanalytical performance with the CNT preparation and the dispersing agent used. Two analytes were tested, showing that a favorable amperometric detection of $\mathrm{NADH}$ and hydrogen peroxide was observed using one kind of CNT in connection to DMF-surface dispersion. $\mathrm{Li}$ and $\mathrm{Ye}^{20}$ reported a systematic study of the electrochemical performance of electrodes prepared with CNTs with different diameters, lengths and functional groups. They demonstrated that the resulting electrodes exhibited remarkably different electrochemical reactivities towards redox molecules such as NADH and ferricyanide, and concluded that CNTs with carboxylate groups showed better electron transfer rates due to the hydrophilic carboxylate groups. They also showed that the length of CNTs affected the electrochemical reactivity, and the long ones had better electrochemical catalysis. Finally in 2012 we evaluate the influence of chemical oxidation on the electrochemical behavior against hydrogen peroxide of long and short multi-walled carbon nanotubes (MWCNT). The results revealed that electrodes modified with pristine short CNT (S-NC) present higher amperometric response against hydrogen peroxide than that obtained using long CNT (L-NC), which correlates with the greater degree of packing observed for L-NC by SEM and the long and thin structures observed in S-NC. On the other hand, the chemical oxidation process increases slightly the sensitivity of resulting electrodes ${ }^{21}$.

Taking into account all the above described points, the main goal of this work was to perform a systematic study on the effects of the nature of nanotubes with regard to the electroanalytical biosensing properties of modified glassy carbon electrodes. To this end, CNTs of two different lengths were used that were subjected to a standard chemical oxidation process for 3 and $6 \mathrm{~h}$. Chitosan $(\mathrm{CHI})$ was selected as a dispersing agent because it possesses a high permeability and is a good matrix for biomacromolecule immobilization, which has facilitated its wide use in electroanalysis ${ }^{22-24}$. Pauliukaite et al. conducted a study on the immobilization effectiveness of functionalized carbon nanotubes into $\mathrm{CHI}$ using different cross-linking agents and tested the modified electrodes for the determination of hydrogen peroxide. Additionally, in 2007 we reported ${ }^{25}$ on the analytical performance of glassy carbon electrodes (GCEs) modified with a dispersion of CNTs in CHI for the quantification of DNA.

In this context, dsDNA was selected as analytical probe, since CNTmodified electrodes have been widely used to quantify DNA, to evaluate DNA damage and for hybridization studies ${ }^{25-28}$. We conclude that the GCE/CNT response strongly depends on the length of CNTs, their degree of oxidation.

\section{MATERIAL AND METHODS}

\subsection{Reagents}

CNTs of two lengths were used: Short CNTs (1-5 um long and $30 \pm 15$ $\mathrm{nm}$ diameter), termed S-NCs, and long CNTs (5-20 $\mu \mathrm{m}$ long and $30 \pm 15 \mathrm{~nm}$ diameter), termed L-NCs. Both types of CNTs were provided by NanoLab (USA).

To compare the effects of chemical treatment, a small fraction of the CNTs were oxidized by $3 \mathrm{M} \mathrm{H}_{2} \mathrm{SO}_{4} / \mathrm{HNO}_{3}$ (3:1) solutions by refluxing. A fraction of the CNTs were oxidized for $3 \mathrm{~h}$ (i.e., S-NCO3 and L-NCO3), while another fraction was oxidized for $6 \mathrm{~h}$ (i.e., S-NCO6 and L-NCO6). Finally, oxidized CNTs were filtered and washed thoroughly with deionized water until a neutral $\mathrm{pH}$ was reached, and then the CNTs were dried at $50^{\circ} \mathrm{C}$ for $24 \mathrm{~h}$. The physical and chemical characterization of the samples was previously informed by us 29 .

CHI of medium molecular weight (Cat. $\left.\mathrm{N}^{\circ} 44887-7\right)$ was obtained from Aldrich, glutaraldehyde (GTA) $(25 \% \mathrm{~V} / \mathrm{V}$ aqueous solution) was purchased from Baker and hydrogen peroxide ( $30 \% \mathrm{~V} / \mathrm{V}$ aqueous solution) was purchased from Merck.

Calf thymus dsDNA (activated and lyophilized, Cat. No 4522) was from Sigma. Stock solutions of dsDNA (1000 mg/L) were prepared with Tris-EDTA buffer (20 mM Tris-HCl, 1 mM EDTA, pH 8.0).

All solutions were prepared with ultrapure water $(\rho=18 \mathrm{M} \Omega)$ from a Millipore-MilliQ system (MQ water). A buffer solution containing $0.2 \mathrm{M}$ formate, $\mathrm{pH} 5.00$ (FBS), was employed as a supporting electrolyte for DNA studies. All chemicals were used as received. 


\subsection{Apparatus}

Differential pulse voltammetric (DPV), and amperometric measurements were performed with a CHI 440 setup (CH Instruments Inc., USA). A GCE of $3 \mathrm{~mm}$ diameter (Model $\mathrm{CHI} 104, \mathrm{CH}$ Instruments) was used as a bare working electrode. A platinum wire and $\mathrm{Ag} / \mathrm{AgCl}, 3 \mathrm{M} \mathrm{NaCl}$ (BAS, Model RE-5B) were used as the counter and reference electrodes, respectively. All potentials are given relative to the reference electrode. A magnetic stirrer provided convective transport when necessary. CNT)

\subsection{Preparation of CNT-modified glassy carbon electrode (GCE/CHI-}

CNTs were dispersed in a $1.0 \% \mathrm{~W} / \mathrm{V}$ CHI solution prepared in $1.0 \% \mathrm{~V} / \mathrm{V}$ acetic acid solution by sonicating for 30 min with a final concentration of 1 $\mathrm{mg} \mathrm{mL}{ }^{-1}$. Prior to surface modification, the GCE was cleaned by polishing with 0.3 and $0.05 \mu \mathrm{m}$ alumina slurries for $1 \mathrm{~min}$. The immobilization of CNTs was performed by casting the GCE with $8 \mu \mathrm{L}$ of the CHI-CNT dispersion. Optimum conditions included drying the dispersion that was dropped onto the GCE for $15 \mathrm{~min}$ at $50{ }^{\circ} \mathrm{C}$, followed by reaction with $3.0 \%$ V/V GTA for $2 \mathrm{~s}$. The electrode was then washed by immersion in buffer solution ( $0.20 \mathrm{M}$ PBS $\mathrm{pH}$ 7.40) for $10 \mathrm{~s}$

\subsection{SECM Experiments}

The experiments were carried out in $0.20 \mathrm{M}$ PBS pH 7.40, using ferrocenemethanol $(\mathrm{FcOH})$ as the redox mediator. The tip potential was held at $0.500 \mathrm{~V}$ to produce oxidation of $\mathrm{FcOH}$, while the substrate potential was held at $0.000 \mathrm{~V}$ to permit feedback between the electrodes. $\mathrm{FcOH}_{\mathrm{ox}}$ generated at the tip was reduced at this potential, thus regenerating the parent $\mathrm{FcOH}$.

Part of the CHI-CNT film (no more than $1 / 3$ of the electrodic surface) was removed and an approach curve was conducted on the exposed glassy carbon surface. The tip scan rate was $0.5 \mu \mathrm{m} \mathrm{s}^{-1}$. The tip was stopped when the experimental feedback current $\left(i_{\mathrm{T}}\right)$ reached 1.25 times the value of $i_{\mathrm{T}, \infty}$ $\left(i_{\mathrm{T}, F}=4 n F D C a\right.$, where $F$ is the Faraday constant, $n$ is the number of electrons transferred in the tip reaction, $D$ is the diffusion coefficient of the electroactive species, $C$ is the bulk concentration of the species and $a$ is the tip radius). According to the theoretical curve that describes the dependence of $i_{\mathrm{T}}$ on the distance between the tip and the substrate $d, 1.25$ times $i_{\mathrm{T}, \infty}$ corresponds to a $d$ $\approx 10 \mu \mathrm{m}$ when a $5 \mu \mathrm{m}$ tip radius is used ${ }^{30}$.

After the approach curve, the tip was moved in the $x$-direction to confirm that the tip was over the film, and a series of constant height $100 \mu \mathrm{m} \times 100 \mu \mathrm{m}$ area SECM images were recorded at a tip scan rate of $1 \mu \mathrm{m} \mathrm{s}^{-1}$. The results are presented in the dimensionless form of $\mathrm{I}_{\mathrm{T}}$ by normalizing the $i_{\mathrm{T}}$ by the steadystate current obtained when the tip was far from the substrate $\left(i_{\mathrm{T}, \Psi}\right)$, i.e., $\mathrm{I}_{\mathrm{T}}=i_{\mathrm{T}} /$ $i_{\mathrm{T}, \Psi}$

\subsection{Cyclic voltammetry}

The cyclic voltammetric experiments were performed using $0.10 \mathrm{M}$ Britton-Robinson buffer solution, $\mathrm{pH} 7.40$, as the supporting electrolyte. All experiments were conducted at room temperature by varying the sweep rate between 0.010 and $0.300 \mathrm{~V} \mathrm{~s}^{-1}$. The experiments were conducted in triplicate.

\subsection{Electrochemical detection of DNA}

DNA adsorption: The given electrode was immersed in a stirred supporting electrolyte solution containing $45 \mathrm{mg} / \mathrm{L}$ of DNA, and accumulation was allowed at open circuit potential for a given time. The electrode containing the adsorbed DNA layer was washed for $10 \mathrm{~s}$ with buffer solution and transferred into a buffer solution in which a DPV run was performed. The experiments were conducted in quadruplicate.

DPV operating conditions: potential increment $0.004 \mathrm{~V}$, pulse amplitude $0.05 \mathrm{~V}$, and pulse period $0.2 \mathrm{~s}$. The anodic current was around $1.0 \mathrm{~V}$, which corresponded to the guanine oxidation that was used as an analytical signal.

\section{RESULTS AND DISCUSSION}

\subsection{Electrochemical characterization of GCE/CHI-CNT electrodes}

3.1.1. Cyclic voltammetry

The modified electrodes using short (S-NC, S-NCO3 and S-NCO6) and long (L-NC, L-NCO3 and L-NCO6) CNTs were first characterized by cyclic voltammetry in $0.10 \mathrm{M}$ Britton-Robinson buffer, $\mathrm{pH}$ 7.40. The experiments showed that capacitive currents recorded between -0.6 and $0.8 \mathrm{~V}$ increased with the sweep rate, and no faradic currents were observed (data not shown). Therefore, the capacitance, as reflect of the electroactive areas of the resulting electrodes, was calculated from plots of the current recorded at $0.2 \mathrm{~V}$ against the scan rate $\left(0.010-0.300 \mathrm{Vs}^{-1}\right)^{18}$. The resulting capacitance values of the $\mathrm{GC} /$
CHI-CNT electrodes are: 74.8, 82.1 and $110.0\left(\mu \mathrm{As} \mathrm{V}^{-1}\right)$ for S-NC, S-NCO3 and $\mathrm{S}-\mathrm{NCO} 6$, and 52.9, 60.3 and $68.3\left(\mu \mathrm{A} \mathrm{s} \mathrm{V}^{-1}\right)$ for $\mathrm{L}-\mathrm{NC}, \mathrm{L}-\mathrm{NCO} 3$ and $\mathrm{L}-\mathrm{NCO} 6$, respectively. The resulting capacitances are several times higher than those determined for bare GCE $\left(10.3 \mu \mathrm{A} \mathrm{s} \mathrm{V}^{-1}\right)$ and $\mathrm{CHI}$ modified GCE $(8.4 \mu \mathrm{As}$ $\mathrm{V}^{-1}$ ). Additionally, S-NC-modified electrodes exhibited larger capacitances than L-NC-modified ones, indicating that S-NC-modified electrodes have higher electroactive surfaces which suggests that they will have better performance as electrochemical sensors than L-NC. On the other hand, at longer oxidation times, higher capacitance values were obtained for both short and long CNTs. This result is concordant with the fact that the oxidation process increases oxygen functional groups (i.e., polar groups) and then improves the wettability of the CNT surface in the electrolyte, resulting in a corresponding increase of the real area of the built-up double layer ${ }^{20,30}$.

\subsubsection{Scanning Electrochemical Microscopy}

Using SECM feedback mode ${ }^{29}$ and $\mathrm{FcOH}$ as a redox mediator we evaluate simultaneously the topography and the electroactivity of the modified surfaces on the $\mathrm{mm}$ scale. As was reported previously by us ${ }^{7}$, bare GCE showed a homogeneous surface with a normalized current $\left(\mathrm{I}_{\mathrm{T}}\right)$ of 1.25 and the GCE/CHI surface was homogeneous but less electroactive because a decrease in the $\mathrm{I}_{\mathrm{T}}$ value was observed (0.75). A completely different behavior was observed when CNTs were incorporated onto the electrode. Figure 1 shows SECM images obtained for GCE/CHI-CNTs prepared using short (S-NCO3 and S-NCO6) and long (L-NCO3 and L-NCO6) CNTs. As can be seen, there is a significant difference between the images obtained when short (images A, C) and long (images B, D) CNTs were used. The surfaces obtained with S-NCs were more homogeneous than those obtained using L-NCs. These results indicate that CHI is more efficient as a dispersing agent for shorter CNTs.

However, as the oxidation time increased, surfaces that are more homogeneous were produced. This suggests that the incorporation of oxygen functionalities on the CNT surface ${ }^{28,31}$ allowed for more disaggregation of CNTs, which was probably due to electrostatic interactions with the positively charged CHI.
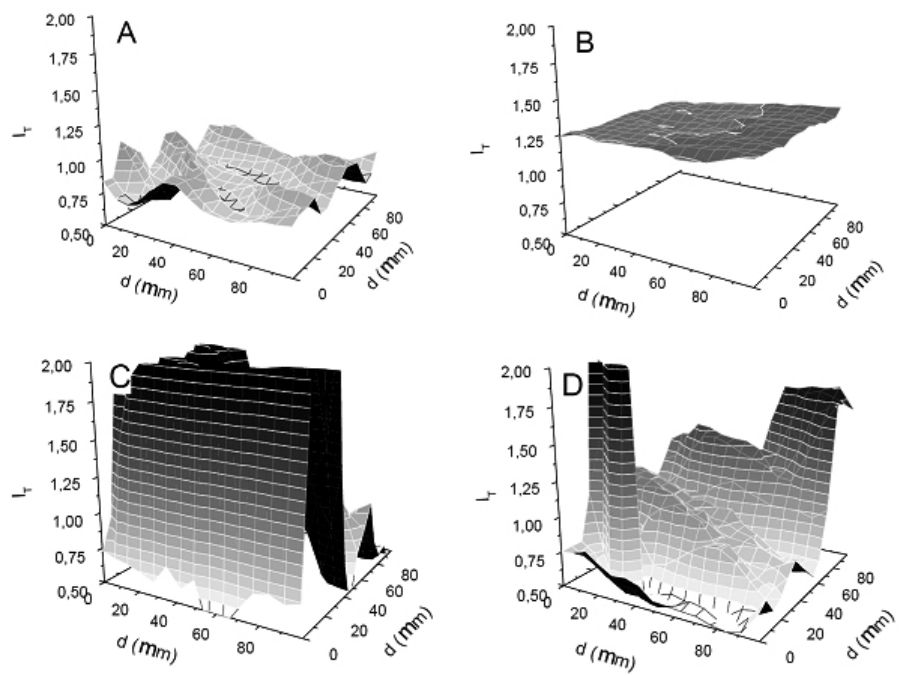

Figure 1. SECM images of GCEs modified with oxidized CNTs dispersed in CHI: (A) S-NCO3 (B) L-NCO3 (C) S-NCO6 and (D) L-NCO6. The redox mediator was ferrocenemethanol. $\mathrm{E}_{\mathrm{T}}=0.500 \mathrm{~V}, \mathrm{E}_{\mathrm{S}}=0.000 \mathrm{~V}$.

\subsection{Electrochemical behavior of dsDNA on GCEs/CHI-CNTS}

3.2.1. Effect of carbon nanotube length.

Following the protocol described previously by Bollo et al. ${ }^{24}$ for GCEs/ CHI-S-NCs, start evaluating the electrochemical response of dsDNA using pristine CNT modified electrodes (GCEs/CHI-sNCs and GCEs/CHI-L-NCs electrodes). Figure 2a shows a DP voltammograms for $45 \mathrm{mg} / \mathrm{L}$ dsDNA obtained after 4 min of accumulation time at open circuit potential using S-NCs (solid lines) and L-NCs (dotted lines). In both cases, a signal corresponding to guanine oxidation was observed. The highest peak current was obtained using GCEs/CHI-L-NCs; however, the peak potential was also higher than that observed using GCEs/CHI-S-NCs (1.000 V vs. $0.940 \mathrm{~V}$, respectively), indicating that the guanine oxidation was slightly less favorable to occur on 
L-NC-modified electrodes. Clearly, the appearance of an oxidation signal around $1.0 \mathrm{~V}$ allowed us to conclude that it is plausible to build modified electrodes using long CNTs to adsorb dsDNA and to detect it through guanine oxidation.

Independent of the size of CNTs used, higher accumulation times produced an increase in the current intensity of the dsDNA signal, with maximum intensities at $4 \mathrm{~min}$ (Fig. 3a). This effect could be attributed to a favored adsorption process provided by the interaction between the negatively charged dsDNA and the amine groups of $\mathrm{CHI}$ that are positively charged at the working $\mathrm{pH}(5.00)$. However, at all accumulation times tested, the dsDNA oxidation signal was always higher with GCEs/CHI-L-NCs than with GCEs/CHI-S-NCs, by approximately $45 \%$.

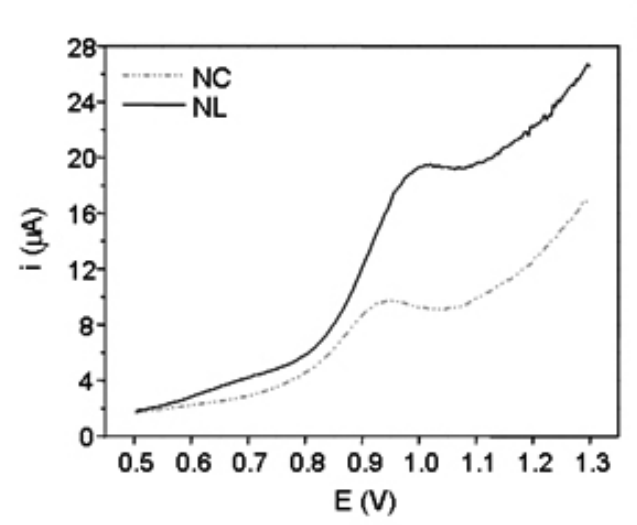

(a)

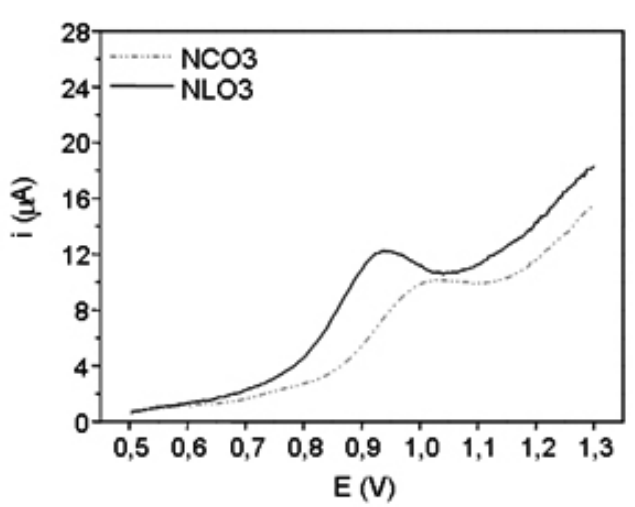

(b)

(c)

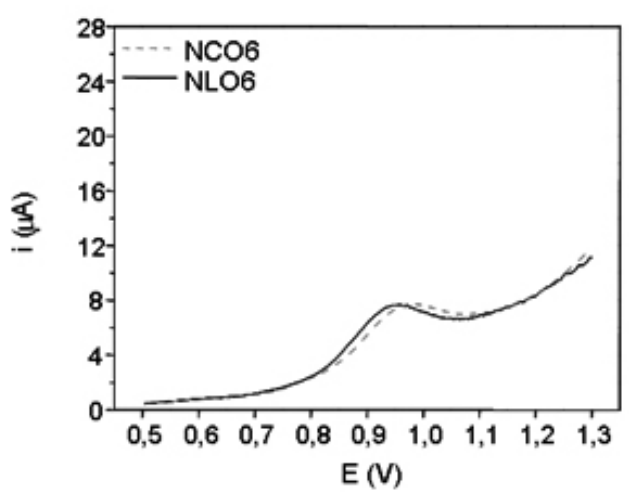

Figure 2. DP voltammograms of $45 \mathrm{mg} / \mathrm{L}$ dsDNA at modified electrodes with different CNTs and 4 min accumulation time: (a) GCE/CHI-S-NC (dotted line) and GCE/CHI-L-NC (solid line), (b) GCE/CHI-S-NCO3 (dotted line) and GCE/CHI-L-NCO3 (solid line) and (c) GCE/CHI-S-NCO6 (dotted line) and GCE/CHI-L-NCO6 (solid line). The experiments were conducted in quadruplicate.

\subsubsection{Effect of the oxidation time of CNTs}

CNTs were oxidized in order to incorporate functional groups such as carboxylic acids on the graphitic surface. Figures $2 b$ and $c$ show DP voltammograms obtained for modified GCEs with CNTs oxidized for 3 and 6 $\mathrm{h}$, respectively. As was previously observed for unoxidized CNTs, the resulting current intensities were greater for longer CNTs when the samples were oxidized $3 \mathrm{~h}$. However when the CNTs were oxidized for $6 \mathrm{~h}$ no significant differences were observed in both current intensity and peak potential.

Comparing all the electrodic surfaces (Fig. 2), a dramatic decrease in dsDNA oxidation response was observed as the CNT oxidation time increased. This decrease was observed using both S-NC and L-NC, and could be explained by two effects: (a) First, the deprotonated - $\mathrm{COO}^{-}$introduced into the structures of CNTs by the oxidation process interact with the amine groups of CHI. This interaction decrease the electrostatic contribution of $\mathrm{CHI}$ for the adsorption process of dsDNA because fewer amine groups are free to interact with the dsDNA. (b) Second, a reduction of the surface area, determined by BET analysis in a previous work ${ }^{20}$, paper that indicates with more compact surfaces when the CNT oxidation time increase leaving less surface area to adsorb DNA.

The oxidation currents (Fig. 3) obtained at several accumulation times using $3 \mathrm{~h}$ oxidized CNTs were higher for L-NCO3 than S-NCO3 by approximately $40 \%$ and when CNTs were oxidized for $6 \mathrm{~h}$, the current intensities were still higher for L-NCO6, but only in about $25 \%$. In addition, in some cases, no significant differences in current values were observed (for example, accumulation times of 4 and 5 min, Fig. 3c).

In conclusion, when the CNT oxidation time was increased, a large fraction of functional groups (mainly $\mathrm{COOH}$ ) was incorporated onto the CNT structure, which improves both dispersion in CHI and electroactivity, as can be seen by SECM and CV. However, at the same time, it produced a decrease in the sensor response against dsDNA because a decreased current was observed.

In all cases, the peak current values of the guanine oxidation signal did not exceed $10 \%$ of RSD for the 4 different electrodes.

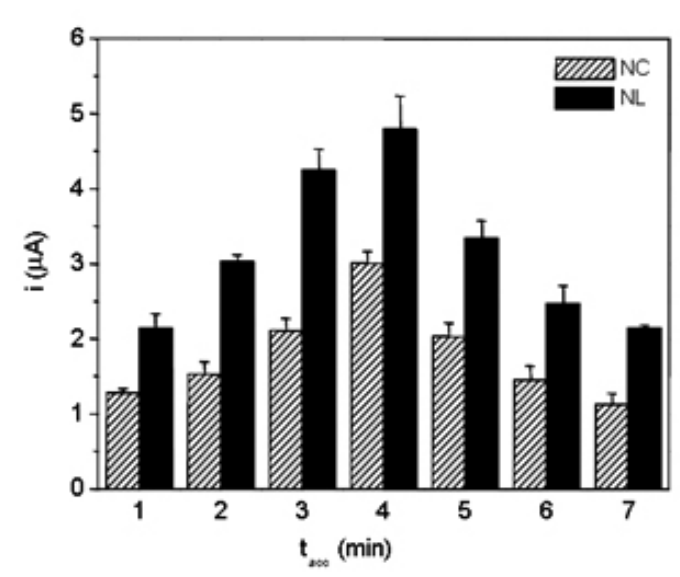

(a)

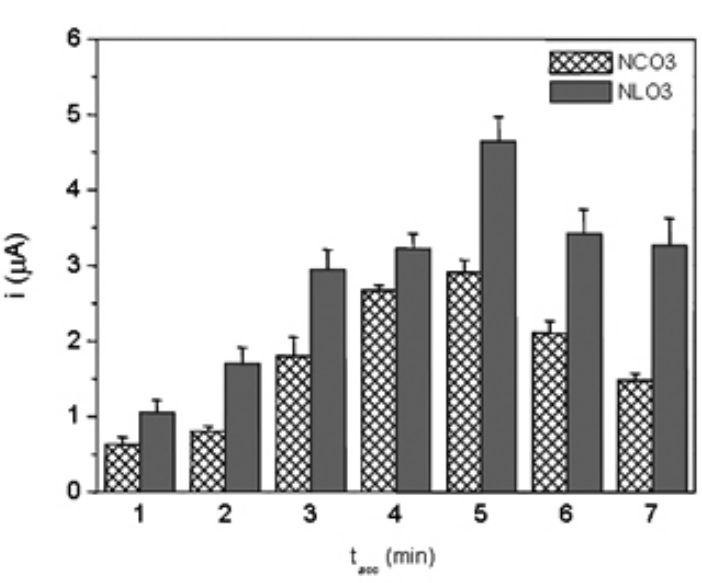

(b) 


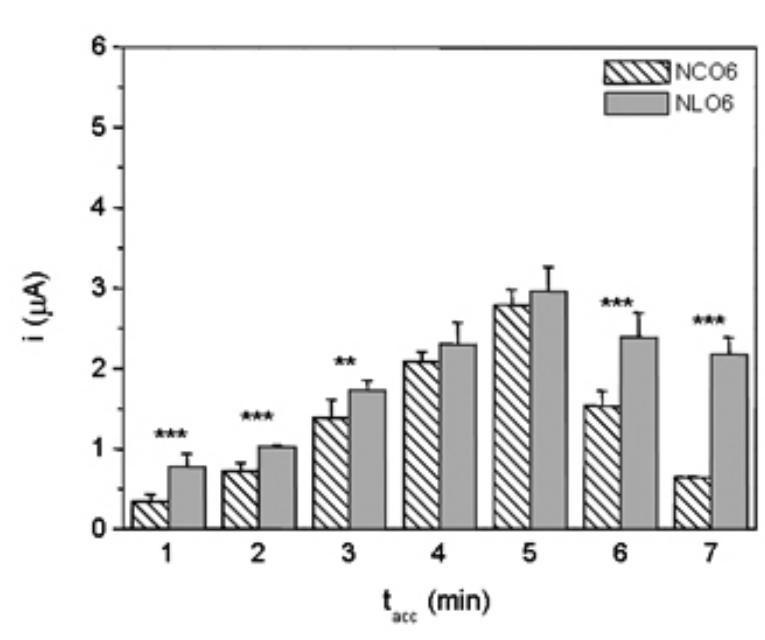

(c)

Figure 3. Current values obtained by DPV at different accumulation times for $45 \mathrm{mg} / \mathrm{L}$ dsDNA: (a) GCE/CHI-S-NC and GCE/CHI-L-NC, (b) GCE/CHIS-NCO3 and GCE/CHI-L-NCO3, (c) GCE/CHI-S-NCO6 and GCE/CHI-LNCO6. [(***) p value $<0.001,(* *) \mathrm{p}$ value $<0.01]$ The experiments were conducted in quadruplicate.

\section{CONCLUSIONS}

The electrochemical sensing properties of CNTs not only depend on the structure of the CNTs, such as length and oxidation state, but also the chemical nature of the analyte. Although the experiments here showed an increase in the capacitance of oxidized CNTs independent of the length of each type of $\mathrm{CNT}$, a remarkable difference was obtained in the final sensing properties of the resulting electrodes. Thus, short CNTs were more efficient in the electrooxidation of hydrogen peroxide, which could be associated with better diffusion of small analytes to the CNT surface and a more efficient electron transfer process. A dramatic difference was observed when the analyte was a biopolymer such as dsDNA, where the most efficient electrodic surface was found with long CNTs. Moreover, the oxidation process had a negative correlation with the detection of dsDNA. In this case, the total surface area seemed to be the most important parameter to be considered for developing an efficient genosensor. Therefore it is not possible a priori to predict which $\mathrm{CNT}$ will be the best for detecting or quantifying a particular chemical species.

\section{ACKNOWLEDGEMENTS}

Financial support from the Fondecyt-CHILE (Grant 1120246) is gratefully acknowledged. P.C. acknowledges CONICYT scholarships for PhD studies in Chile, PhD scholarship support (AT-24091012) and PhD fellowship study abroad (BECAS-CHILE).

\section{REFERENCES}

1. P. Yanez-Sedeno, J. Riu, J. M. PingarronF. X. Rius, Trac-Trends in Analytical Chemistry, 29, 939, (2010)

2. S. K. Vashist, D. Zheng, K. Al-Rubeaan, J. H. LuongF. S. Sheu, Biotechnology Advances, (2010)

3. V. K. Gupta, R. Jain, K. Radhapyari, N. JadonS. Agarwal, Analytical Biochemistry, 408, 179, (2011)

4. Z. A. Alothman, N. Bukhari, S. M. WabaidurS. Haider, Sensors and Actuators B-Chemical, 146, 314, (2010)

5. B. RezaeiS. Damiri, Journal of Hazardous Materials, 183, 138, (2010)

6. L. Vaisman, H. D. WagnerG. Marom, Advances in Colloid and Interface Science, 128, 37, (2006)

7. Z. Z. Li, Z. Y. WuK. Li, Analytical Biochemistry, 387, 267, (2009)

8. R. Rastogi, R. Kaushal, S. K. Tripathi, A. L. Sharma, I. KaurL. M. Bharadwaj, Journal of Colloid and Interface Science, 328, 421, (2008)

9. J. Amiran, V. Nicolosi, S. D. Bergin, U. Khan, P. E. LyonsJ. N. Coleman, Journal of Physical Chemistry C, 112, 3519, (2008)

10. K. S. Liao, A. Wan, J. D. BatteasD. E. Bergbreiter, Langmuir, 24, 4245, (2008)
11. V. Georgakilas, A. Bourlinos, D. Gournis, T. Tsoufis, C. Trapalis, A. Mateo-AlonsoM. Prato, Journal of the American Chemical Society, 130, 8733, (2008)

12. E. C. Landis, K. L. Klein, A. Liao, E. Pop, D. K. Hensley, A. V. MelechkoR. J. Hamers, Chemistry of Materials, 22, 2357, (2010)

13. D. Silambarasan, K. Iyakutti, V. Vasu, Chemical Physics Letters 604, 83, (2014)

14. M. Merisalu, J. KruusmaC. E. Banks, Electrochemistry Communications, 12, 144, (2010)

15. I. D. Rosca, M. M. Barsan, I. S. ButlerJ. A. Kozinski, Carbon, 47, 2552, (2009)

16. D. Tasis, N. Tagmatarchis, A. BiancoM. Prato, Chemical Reviews, 106, $1105,(2006)$

17. L. J. Meng, C. L. FuQ. H. Lu, Progress in Natural Science, 19, 801, (2009)

18. J. Li, A. Cassell, L. Delzeit, J. HanM. Meyyappan, Journal of Physical Chemistry B, 106, 9299, (2002)

19. N. S. Lawrence, R. P. DeoJ. Wang, Electroanalysis, 17, 65, (2005)

20. X. L. LiJ. S. Ye, Electroanalysis, 20, 1917, (2008)

21. P. Cañete-Rosales, V. Ortega, A. Álvarez-Lueje, S. Bollo, M. González, A. AnsónM. T. Martínez, Electrochimica Acta, 62, 163, (2012)

22. M. G. Zhang, A. SmithW. Gorski, Analytical Chemistry, 76, 5045, (2004)

23. L. Rassaei, M. SillanpaaF. Marken, Electrochimica Acta, 53, 5732, (2008)

24. R. Pauliukaite, M. E. Ghica, O. FatibelloC. M. A. Brett, Analytical Chemistry, 81, 5364, (2009)

25. S. Bollo, N. F. FerreyraG. A. Rivas, Electroanalysis, 19, 833, (2007)

26. T. Yang, N. Zhou, Y. C. Zhang, W. Zhang, K. JiaoG. C. Li, Biosensors and Bioelectronics 24, 2165, (2009)

27. T. T. N. Lien, T. D. Lam, V. T. H. An, T. V. Hoang, D. T. Quang, D. Q. Khieu, T. Tsukahara, Y. H. LeeJ. S. Kim, Talanta, 80, 1164, (2010)

28. X. Q. Ran, R. Yuan, Y. Q. Chai, C. L. HongX. Q. Qian, Colloids and Surfaces B-Biointerfaces, 79, 421, (2010)

29. P. Cañete-Rosales, V. Ortega, A. Alvarez-Lueje, S. Bollo, M. González, Ansón, A., Martínez, M.T, Electrochimica Acta, 62 13, (2012)

30. J. Bard, F.F. Fan, J. Kwak, L. Ovadia, Analytical Chemistry, 61, 132, (1989)

31. C. Li, D. Wang, T. Liang, X. WangL. Ji, Mater. Lett., 58, 3774, (2004)

32. F. C. Moraes, M. F. Cabral, L. H. MascaroS. A. S. Machado, Surface Science, 605, 435, (2011) 